\title{
Comparative analysis on Void Node Removal Routing algorithms for Underwater Wireless Sensor Networks
}

\author{
Mukhtiar Ahmed
}

Quaid-e-Awam University, of Science and Technology, Nawabshah, Sindh, Pakistan
Fauzia Talpur,

Department of Computer

Science, Faculty of Computing

UTM, Malaysia
M.Ali Soomro

Quaid-e-Awam University, of Science and Technology, Nawabshah, Sindh, Pakistan

\begin{abstract}
The designing of routing algorithms faces many challenges in underwater environment like: propagation delay, acoustic channel behaviour, limited bandwidth, high bit error rate, limited battery power, underwater pressure, node mobility, localization 3D deployment, and underwater obstacles (voids). This paper focuses the underwater voids which affects the overall performance of the entire network. The majority of the researchers have used the better approaches for removal of voids through alternate path selection mechanism but still research needs improvement. This paper also focuses the architecture and its operation through merits and demerits of the existing algorithms. This research article further focuses the analytical method of the performance analysis of existing algorithms through which we found the better approach for removal of voids.

Keywords: Deployment; voids; water-current; v-shape; depth-adjustment; harsh
\end{abstract}

\section{INTRODUCTION}

In underwater environment the void node removal is one of the major issues which reduce the packets success ratio. The underwater obstacles and depletion of node energy creates the void node along the active packets forwarding path [1-3]. A number of factors individually or a combination of them, caused the void phenomena, such as sparse topology, temporary obstacles, and unreliable nodes or links [4, 5]. Existing relevant routing algorithms based on void node removal are briefly discussed.

\section{RELATED WORK}

Reliable and Energy Balanced Routing Algorithm (REBAR) is proposed by Chen, et al. [6]. In REBAR the two energy models are used to reduce the energy consumption of the ordinary sensor node; one is sphere energy depletion and other is extended energy depletion. REBAR is location based routing protocol, in REBAR the center sink node is placed on water surface, and sensor nodes are deployed in underwater. In REBAR data forwarding mechanism is based on hop-byhop and transmission range $\mathrm{R}$ is fixed between sensor nodes. REBAR is based on the size of broadcast which is the stern alarm of REBAR which consumes the high energy. With high broadcast the more energy will be consumed by sensor nodes and with low broadcast size less energy will be consumed; with these both issues the REBAR keeps the balanced broadcast size mechanism to balance the energy level of the sensor node. In REBAR the distance $\mathrm{d}$ and vector $\mathrm{v}$ are the parameters which are stored in the packets format for calculating the routing direction with distance and vector between source and sink node for data forwarding. The packets information in REBAR is stored in the buffer of every node and on arrival of duplicate packets the node will drop the duplicate packets. The threshold value is used in between source and sink node to ensure the packets forwarding mechanism with right direction. For removal of void regions the boundary-set and non-boundary set mechanisms are adapted, with boundary set the information will be shared by the nodes for the presence of the void regions and alternate path selection mechanism will be adapted for data forwarding. In non-boundary set mechanism the data forwarding mechanism behaves normally [6]. REBAR focuses the data delivery ratio increases with respect to node movement but in real scenario the enhancement of data delivery ratio is not

possible because the nodes behavior in underwater environment is not controllable. Horizontal and vertical node movement methodology is not clearly defined; so obviously packets drop ratio increases and results into the reduction of the overall network throughput. Removal of void regions are just hypothesis and this may also reduce the data delivery ratio.

Vector-Based Void Avoidance (VBVA) routing protocol is proposed by Xie, et al. [7]. VBVA functionality is adapted from VBF with variations for removal of void regions. For removal of void regions the VBVA adapted two mechanisms one is vector-shift and other is back-pressure. Void node can be detected by considering the example as shown in Figure 1(a); $\mathrm{S}$ is source node and $\mathrm{D}$ is destination node. If $\mathrm{S}$ and $\mathrm{T}$ are the start and end points then (ST) $\overrightarrow{ }$ is the forwarding vector of the packets. The nodes $\mathrm{K}, \mathrm{L}$, and $\mathrm{P}$ are advances nodes on the forwarding vector and are denoted by AK, AL, and AP respectively. The nodes have property to detect the presence of a void to listen in the transmission of the packets by its neighbor nodes. The nodes also have a property to record the position information of the forwarding node. In Figure 1 (a) the $\mathrm{K}$ and $\mathrm{L}$ have smaller advances than $\mathrm{P}$ on forwarding vector $(\mathrm{ST}) \overrightarrow{ }$. Node $\mathrm{P}$ has a larger advance among the neighbor nodes within the forwarding pipe and it concludes that it is a void node. Node $\mathrm{P}$ detects the void on a current forwarding vector and tries to bypass the void by changing the forwarding vector of the packets through the alternate route. To bypass the void through alternate route the node $\mathrm{P}$ can adapt vector-shift and back-pressure for removal of void region. In vector-shift mechanism the boundary of convex void by shift technique is adapted for data forwarding.

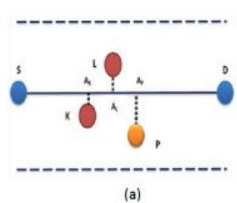

Figure 1: (a)Void node detection mechanism, (b) Vector shift,
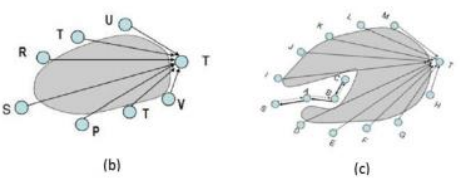

\section{(c) Back pressure [7]}


In Figure 1(b) the dashed area is void area whereas $S$ is sender node and $\mathrm{T}$ is destination node. Data forwarding mechanism starts from $\mathrm{S}$ to $\mathrm{T}$ along vector $(\mathrm{ST}) \overrightarrow{ } \rightarrow$ if neighbor nodes of $\mathrm{S}$ like: $\mathrm{P}$ and $\mathrm{R}$ are not within the range in forwarding pipe then no forwarding mechanism will be considered. During the transmission mechanism if sender node $S$ is unable to hear any movement from the acoustic channel than $\mathrm{S}$ node will consider the occurrence of void region and node $\mathrm{S}$ will forward the vector-shift control packet to the neighbor nodes to change the current forwarding vector position. In Figure 1(c) the shadow area is the concave void and node $S$ is sender node whereas node $\mathrm{T}$ is sink node. If node $\mathrm{S}$ forwards the packets to node $\mathrm{C}$ and $\mathrm{C}$ is unable to forward the packets then node $\mathrm{C}$ will broadcast the back-pressure control packet to node B. Node B will also broadcast the back-pressure control packet to route the packet for node A. Node A will broadcast for node $\mathrm{S}$, finally node $\mathrm{S}$ will shift the forwarding vector to node $(\mathrm{HT}) \overrightarrow{ }$ and (DT) $\overrightarrow{ }$. The vector-shift mechanism is used to forward the packets towards sink node. The continuous node movement affects the performance of VBVA because through continuous movement node may away from the virtual pipe and will drop the packets. VBVA has adapted the vector-shift and back-pressure mechanisms without any consideration of the underwater parameters which shows that the authors have just focused the hypothesis. It is also observed that the performance evaluation of the VBVA is only based on VBF.

Hydraulic Pressure Based Any cast Routing (HydroCast) is proposed by [8]. Distributed localization HydroCast is geographic routing. The measured pressure mechanism is used by HydroCast for data forwarding to surface buoys. HydroCast resolves the issues of DBR routing protocol. The depth information with relevant clusters is adapted in HydroCast through pressure levels. The clusters formation mechanism of HydroCast is based on terminal nodes. The cluster formation mechanism is based on calculation of maximum progressive nodes which are nearer to destination nodes. Maximum progressive node plays a vital role in data forwarding mechanism and it has higher priority as compare to other neighbor nodes. For data forwarding the short timeout period is set by HydroCast. Limited flooding approach is used by HydroCast through maximum recovery technique. Flooding mechanism uses the performer or local maximum node for data forwarding. In HydroCast for local maximum node identification the tetra horizontal method is used. The data forwarding mechanism has been adapted between local maximum nodes through limited number of hops. For removal of void regions the greedy approach is used. The multiple numbers of data packets are received by sink node increases the extra burden on network. The energy efficient parameter in HydroCast is not clearly defined.

Depth Controlled Routing (DCR) proposed by Coutinho, et al. [9] is based on centralized algorithm to overcome the communication void problem. The algorithm determines which node become failing in greedy geographic forwarding task and then will calculate the new depth for possible routing. DCR is topology control geographic routing that considers the node mobility to adjust its depth, guaranteeing connectivity and eliminating communication void regions. In DCR the sensor nodes sense the underwater environment and periodically send the collected data towards sonobuoys. The distance of neighbors is considered to its nearest sonobuoys, obtained from beacons in the process of next-hop selection. Thus the neighbor that will be selected to act as a next-hop in the forwarding process is the node which is closest to some sonobuoys among all neighbors. If the selected node to act as next-hop cannot continue with greedy forwarding; it broadcast message to inform its neighbors for its void node situation. The neighbors then update its routing table, removing its void node entry. DCR faces some serious problems like: in sparse area the performance of DCR is reduced. The water pressure and water current affects the forwarder node along active path. No any proper mechanism is defined for removal of void node by DCR.

Void-Aware Pressure Routing (VARP) is proposed by Noh, et al. [10] which uses the local opportunist directional forwarding for data success ratio even in presence of voids. It uses the soft-state breadcrumb approach for mobile networks. It is based on enhanced beaconing and opportunist directional data forwarding. The $\mathrm{V}$-shape architecture for data forwarding and in V-shape if any void region occurs is called trap area. If trap area appears the packets will be forwarded towards the new route through next-hop forwarding mechanism. It is observed that when nodes become spars the performance of VARP become slow due to affected of V-shape by underwater pressure. In VARP, the depth controlling mechanism is also not defined by authors.

Geographic Depth Adjustment Routing (GEDAR) is proposed by Coutinho, et al. [11] which is based on depth adjustment topology controlling mechanism for removal of void nodes. It moves the void node in new depth with greedy forwarding strategy. The sea swarm architecture is adapted for packets forwarding. The sensor nodes are equipped with buoyancybased depth adjustment which adjusts the depth of the sensor nodes in underwater. The depth adjustment mechanism for sensor node location information is based on vertical movement, energy cost values and periodic beaconing. The data packets are forwarded by qualified neighbor node through next-hop forwarder mechanism from source to sink. During packets forwarding mechanism each node will observe the void node and if void node appears during packets forwarding the void node will be shifted towards new depth through greedy forwarding mechanism with two-hop steps. The topology control mechanism defined by GEDAR is not so easy due to water pressure and continuous node movement. The depth calculation mechanism is not properly defined by proposed algorithm.

Opportunist Void Avoidance Routing (OVAR) is proposed by Ghoreyshi, et al. [12] which use the prioritizing the group of candidate nodes with highest packets advancement mechanism. Given the density of neighbor nodes, each forwarding node is able to hold a trade-off between packet advancement and energy consumption by adjusting the number of nodes in its forwarding set. OVAR is also able to select the forwarding set in any direction from the sender without including any hidden node. The operation of OVAR is based on four phases. In first phase an adjacency graph is constructed at every node and using a heuristic some clusters i.e clique sub-graphs is created to ensure that hidden nodes are removed from forwarding sets. In second phase the best forwarding set is selected using expected packets advancement to maximize the chance of successful delivery of packets. In third phase the number of forwarding nodes in the forwarding set is adjusted to make a trade-off between reliability and energy consumption. In fourth phase the holding time is calculated at each candidate node before the forwarding node. In OVAR the removal of void node 
mechanism is not properly defined, if any node becomes as a void node due to underwater obstacles and that node is the packets forwarder node then that node will drop the packets and will reduce the data success ratio.

Void Handling Geo-Opportunist Routing (VHGOR) proposed by Kanthimathi [13] is based on quick hull algorithm for convex or concave voids. When the node approaches a convex void; reconstruction of convex hull helps to determine an alternative way to resume the greedy forwarding if the neighbor within its proximity. Failure of convex void handling during communication void makes VHGOR switch to concave void handling or recovery mode to recover the packets from local maximum node and route the packets towards destination. For convex void handling the immediate forwarder node will sent back ACK to source node within some certain time period; if ACK not received by source node means forwarder node is in void region and VHGOR will manage the alternate route to forward packets through convex hull. During creation of void node; if convex hull will not to be built then VHGOR consider the concave hull or recovery mode. In concave hull the packets are re-routing along the recovery path. The recovery path works from down-stream to upstream to route packets towards destination from alternative paths. VHGOR has adapted the void problem from VBVA and VHGOR is failure to calculate the water depth from sea surface to bottom. No proper node mobility model is defined for controlling of node movement. The multi-hop technique from top to seabed is used; so due to long distance multi-hop technique cannot shows the better results for data success rate and also maximizes the end-to-end delay.

\section{PERFORMANCE ANALYSIS}

In Table 1, the parametric performance analysis of void node removal routing algorithms are shown. The void node removal is based on: node mobility controlled, hop-byhop/end-to-end delay, single/multiple sink, multipath, hello/control packet, and void node removal technique. The analysis is based on protocol operation with packets forwarding mechanism.

\section{CONCLUSION}

Void node removal means the node which may come in underwater obstacles or the node may become dead due to energy depletion. Almost the existing routing algorithms focuses the underwater obstacle and when node becomes void, the node may drop the packets and will affect the overall performance of the network. The REBAR routing algorithm is based on boundary set mechanism to remove the void but it is observed that boundary set approach is not suitable for underwater environment. In same way the HydroCast is based on flooding mechanism for removal of voids, the approach used by Hydrocast is not mentioned in its research paper that how it forwards the packets when node becomes void. From the aforementioned void node algorithms the quick-hull and depth-adjustment mechanisms used by VHGOR and GEDAR are observed the best approaches for removal of voids from underwater environment.

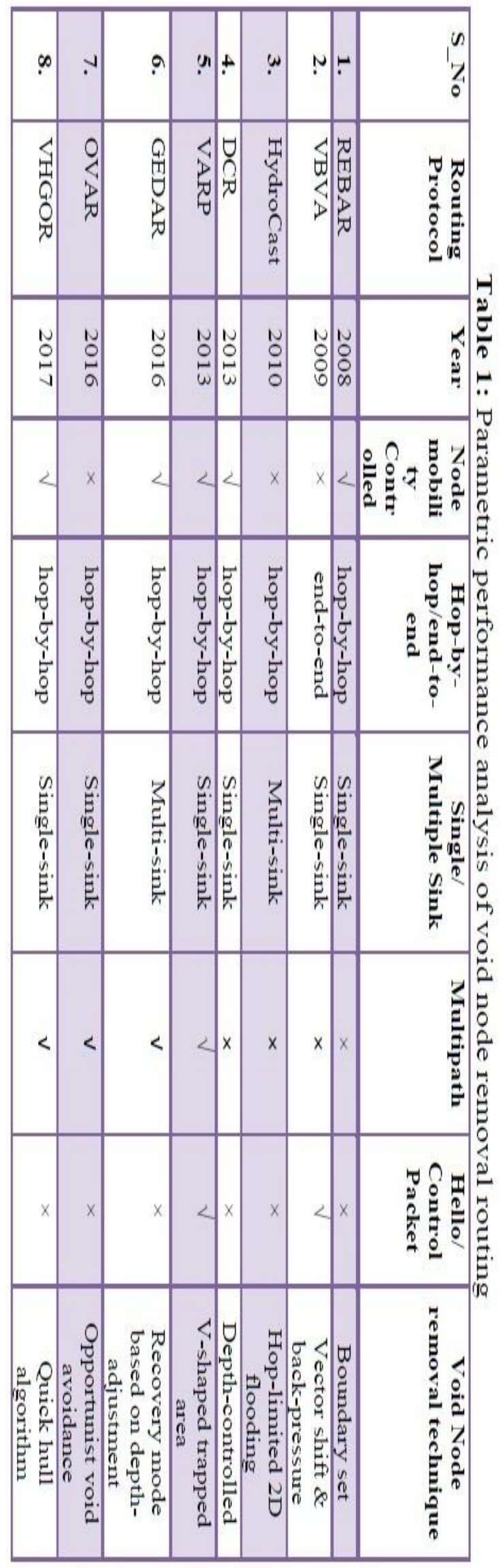




\section{REFERENCES}

[1] R. H. Rahman, C. Benson, and M. Frater, "Routing Protocols for Underwater Ad Hoc Networks," Oceans, 2012, IEEE Conference, Yeosu-si, Jeollanam-do, South Korea, 2012.

[2] Y. Wang, Y. J. Liu, and Z. W. Guo, "Three-dimensional ocean sensor networks: A survey," Journal of Ocean University of China, vol. 11, pp. 436-450, Dec 2012.

[3] P. Casari and M. Zorzi, "Protocol design issues in underwater acoustic networks," Computer Communications, vol. 34, pp. 2013-2025, Nov 2011.

[4] M. Xu, G. Z. Liu, H. F. Wu, and W. Sun, "Towards Robust Routing in Three-Dimensional Underwater Wireless Sensor Networks," International Journal of Distributed Sensor Networks, vol. 9, pp. 1-15, 2013.

[5] S. M. Ghoreyshi, A. Shahrabi, and T. Boutaleb, "VoidHandling Techniques for Routing Protocols in Underwater Sensor Networks: Survey and Challenges," IEEE Communications Surveys \& Tutorials, vol. 19, pp. 800-827, 2017.

[6] J. M. Chen, X. B. Wu, and G. H. Chen, "REBAR: A Reliable and Energy Balanced Routing Algorithm for UWSNs," In Grid and Cooperative Computing, 2008, GCC'08. Seventh International conference on. IEEE, vol. Shenzhen, China, pp. 349-355, 2008.

[7] P. Xie, Z. Zhou, Z. Peng, J.-H. Cui, and Z. Shi, "Void avoidance in three-dimensional mobile underwater sensor networks," in Wireless Algorithms, Systems, and Applications, ed: Springer, Springer, Berlin, Heidelberg, 2009, pp. 305-314.

[8] U. Lee, P. Wang, Y. Noh, F. Vieira, M. Gerla, and J.-H. Cui, "Pressure routing for underwater sensor networks," in INFOCOM, 2010 Proceedings IEEE, San Diego, Calif, USA, 2010, pp. 1-9.
[9] R. W. Coutinho, L. F. Vieira, and A. A. Loureiro, "DCR: Depth-Controlled routing protocol for underwater sensor networks," in Computers and Communications (ISCC), 2013 IEEE Symposium on, Split, Croatia, 2013, pp. 000453-000458.

[10] Y. Noh, U. Lee, P. Wang, B. S. C. Choi, and M. Gerla, "VAPR: void-aware pressure routing for underwater sensor networks," IEEE Transactions on Mobile Computing, vol. 12, pp. 895-908, 2013.

[11] R. W. Coutinho, A. Boukerche, L. F. Vieira, and A. A. Loureiro, "Geographic and opportunistic routing for underwater sensor networks," IEEE Transactions on Computers, vol. 65, pp. 548-561, 2016.

[12] S. M. Ghoreyshi, A. Shahrabi, and T. Boutaleb, "An opportunistic void avoidance routing protocol for underwater sensor networks," in Advanced Information Networking and Applications (AINA), 2016 IEEE 30th International Conference on, Lens, Switzerland, 2016, pp. 316-323.

[13] N. Kanthimathi, "Void handling using Geo-Opportunistic Routing in underwater wireless sensor networks," Computers \& Electrical Engineering, vol. 127, pp. 1-15, 2017.

[14] B. Karp and H.-T. Kung, "GPSR: Greedy perimeter stateless routing for wireless networks," in Proceedings of the 6th annual international conference on Mobile computing and networking, ACM, Boston, MA, USA, 2000, pp. 243-254.

[15] F. Zhou, G. Trajcevski, R. Tamassia, B. Avci, A. Khokhar, and P. Scheuermann, "Bypassing holes in sensor networks: Load-balance vs. latency," Ad Hoc Networks, vol. 61, pp. 16-32, 2017. 